\title{
Perspectiva \\ Intergeneracional del Juego como Patrimonio Cultural
}

\section{Intergenerational Perspective of the Game as a Cultural Heritage}

\section{Perspectiva Intergeracional da Brincadeira como Patrimonio Cultural}

\author{
Adriana Araya Góchez ${ }^{1}$ \\ Marianella Castro Pérez ${ }^{2}$ \\ María Ester Morales Ramírez ${ }^{3}$
}

\section{Resumen}

En el 2013 la Universidad Nacional y la Universidad de Costa Rica iniciaron la investigación "El juego como patrimonio cultural: percepción de las personas de diferentes generaciones en los cantones de Desamparados y Barva", cuyo objetivo fue analizar el juego como patrimonio cultural, desde la percepción que tienen las personas de diferentes grupos generacionales de ambos cantones. Este escrito presenta los resultados del análisis intergeneracional, al contrastar las diferencias y similitudes relacionadas con las siguientes características del juego: conceptualización, importancia, valores que promueve, lugares donde sucede el juego, personas que lo acompañan y tipos de juegos. El enfoque de investigación fue mixto y el tipo fue descriptivo-explicativo ya que proporciona un sentido de entendimiento al juego como patrimonio. La población participante constó de 170 personas de Desamparados y 158 de Barva, conformada por estudiantes de primaria y secundaria, docentes, personas adultas y adultas mayores y representantes municipales, seleccionados según criterios de edad y rol que desempeñaban. Los datos

1 Bachiller en Ciencias de la Educación con énfasis en Educación Preescolar y Licenciada en Ciencias de la Educación con énfasis en Administración de la Educación No Formal, ambos de la Universidad de Costa Rica. Curadora Encargada de la Sección de Educación del Museo de la Universidad de Costa Rica (museo+UCR).

2 Máster en Administración Educativa. Licenciada en Educación Preescolar. Académica, investigadora y extensionista en el Instituto de Estudios Interdisciplinarios de la Niñez y Adolescencia (INEINA) y docente en la División de Educación Básica del Centro de Investigación y Docencia en Educación de la Universidad Nacional de Costa Rica.

3 Doctora en Educación con Énfasis en Investigación Educativa. Máster en Psicología Industrial y Organizacional. Licenciada en Psicología. Académica en el Instituto de Estudios Interdisciplinarios de la Niñez y Adolescencia (INEINA) del Centro de Investigación y Docencia en Educación de la Universidad Nacional de Costa Rica. 
fueron recolectados con cuestionarios que contenían ítes comunes y que estaban dirigidos con base en el grupo generacional. Los principales resultados refieren que el juego es considerado por todos los grupos generacionales como una actividad placentera, que promueve diversión, entretenimiento, disfrute, alegría, convivencia y socialización. El juego se visualiza como una actividad casi exclusivamente de y para los niños y las niñas, en pocas excepciones mencionan a las personas adultas o adultas mayores como sujetos participantes. Casi para la totalidad de la población, el juego es de suma importancia para el desarrollo integral, para el aprendizaje de conceptos y esencialmente de valores. Llama la atención que el juego no es visualizado como un derecho según lo establece la legislación.

Palabras clave: juego, patrimonio, cultura, generaciones.

\begin{abstract}
In 2013, the National University of Costa Rica and the University of Costa Rica launched the research project titled Games as a Cultural Heritage: Perception of People of Different Generations in the Cantons of Desamparados and Barva. Its objective was to analyze games as a cultural heritage, from the perception people have of different generational groups in both cantons. This paper presents the results obtained from the intergenerational analysis when contrasting differences and similarities related to the following characteristics of games: conceptualization, importance, values promoted, places where games happen, people involved, and types of games. The research approach was a mix of descriptive and explanatory designs because it provides a sense of understanding the game as a heritage. From Desamparados, 170 people took part in the research; and 158 from Barva. They were primary and secondary students, teachers, adult and senior citizens, and municipal agents. The participants were selected following criteria of age and the role they play. Data were collected using questionnaires that contained common items, and that were based on the generational group. The main results show that all age groups consider games as a pleasurable activity, which promotes fun, entertainment, enjoyment, joy, coexistence and socialization. Games are considered as activities almost exclusively for, and practiced by, boys and girls; a few exceptions mentioned adults and older people as participants in games. Almost the whole population considered games as of extreme importance for integral development, and for the learning of concepts and, essentially, of values. It is striking that games are not considered as a right as set out in the Costa Rican legislation.
\end{abstract}

Keywords: games, heritage, culture, generations.

\title{
Resumo:
}

Em 2013, a Universidade Nacional e a Universidade da Costa Rica iniciaram a investigação "A brincadeira como património cultural: percepções das pessoas de diferentes gerações nas comunidades de Desamparados e Barva", cujo objetivo era analisar a brincadeira como património cultural, a partir da percepção das pessoas de diferentes gerações de ambos comunidades. Este artigo apresenta os resultados da análise intergeracional, depois de contrastar as diferenças e semelhanças relacionadas com as seguintes características das brincadeiras: conceituação, importância, valores promovidos, lugares aonde acontece a brincadeira, pessoas que acompanham e tipos de brincadeiras. A abordagem da pesquisa foi mista, do tipo explicativo descritivo, 
pois proporciona um sentido de compreensão da brincadeira como algo próprio. A população participante foi de 170 pessoas de Desamparados e 158 de Barva, composta por estudantes do ensino fundamental e médio, professores, adultos e idosos, representantes municipais, selecionados de acordo com critérios de idade e função que desempenham. Os dados foram obtidos por meio de questionários contendo itens comuns e direcionados com base no grupo etário. Os principais resultados indicam que as brincadeiras são consideradas por todos os grupos intergeracionais como uma atividade prazerosa, promotora de diversão, entretenimento, gozo, alegria, convívio e socialização. A brincadeira é vista como uma atividade quase exclusivamente de e para as crianças, onde a participação das pessoas adultas são exceções. Para grande parte da população, brincar é muito importante para o desenvolvimento integral, para a aprendizagem de conceitos e essencialmente, de valores. Vale ressaltar que brincar não é visto como um direito estabelecido por lei.

Palavras-chave: brincadeira, herança, cultura, gerações.

\section{Introducción}

El presente artículo contiene un extracto de los resultados del proyecto "El juego como patrimonio cultural: percepción de las personas de diferentes generaciones en los cantones de Desamparados y Barva”, específicamente del análisis intergeneracional, contrastando las características del juego relacionadas con las siguientes características del juego: conceptualización, importancia, valores que promueve, lugares donde se efectúa el juego, personas que lo acompañan y tipos de juegos de preferencia. La investigación se llevó a cabo en dos comunidades, el cantón Central de Desamparados en San José y el cantón de Barva en Heredia, en ambas poblaciones se abordó el tema con un grupo de niños y niñas escolares (de tercero y sexto grados), un grupo de jóvenes de secundaria (de octavo y undécimo años), docentes de primaria y secundaria, personas adultas mayores (entre 25 y 50 años de edad), personas adultas (de 50 años en adelante) y representantes del gobierno local de cada provincia.

Países latinoamericanos y europeos establecieron la relevancia de contemplar el juego como patrimonio cultural intangible, ya que cumple con la función de fortalecer la identidad cultural de los pueblos. Específicamente en el ámbito nacional, tanto el Ministerio de Cultura y Juventud, como el Centro de Investigación y Conservación del Patrimonio Cultural, las universidades públicas y los gobiernos locales, entre otros, han puesto en sus agendas institucionales la importancia de redescubrir y valorar lo propio, con el fin de hacer frente a la llamada crisis cultural. Al respecto, Garre, Sanguinetti y Ramos (1997) consideran que "el juego es el espejo de cada civilización" (párr. 1) y sin duda alguna forma parte del acervo cultural de cada comunidad. 
Las investigaciones que se encontraron en el ámbito internacional y nacional brindan un panorama general acerca de la importancia de generar en el país diversas producciones académicas vinculadas con el juego como patrimonio, el rescate y valor en la construcción de las identidades de las comunidades. Por ello, el interés de este artículo es analizar, identificar y caracterizar el juego como patrimonio intergeneracional.

El juego en su función como patrimonio cultural promueve conductas para que los individuos de una sociedad logren adaptarse a los requerimientos sociales-culturales-políticos-económicos y favorece el rescate y resguardo de tradiciones y costumbres, consideradas un bien patrimonial como parte de su identidad. El juego es, a su vez, una actividad común en todas las personas, que rompe fronteras de edad, zona geográfica, nivel socioeconómico, condiciones físicas e intelectuales y se considera como patrimonio cultural al reconstruir y transformar día a día la cultura. Por ello, se parte de la percepción y experiencia que del juego tienen diversos grupos generacionales, para hacer un contraste de vivencias que permitan caracterizar el juego desde la visualización de las relaciones y diferencias en diversas épocas.

Según Jaramillo (2009), todos los seres humanos tienen una serie de creencias y costumbres diferentes según época, cultura, educación, experiencias personales, ejemplos en el hogar, medio y adoctrinamiento moral, religioso o ideológico a que han estado sometidos en su ambiente. Por lo tanto, se dice que "cuanta más cultura exista en un país y se ofrezca mayor oportunidad a las personas para desarrollarse, los individuos de esa sociedad tendrán más posibilidad de aumentar sus aptitudes" (p. 79). Por lo antes expuesto, se consideran valiosos los aportes de este escrito para conocer la percepción de las personas de diversos grupos generacionales en relación con el juego y la cultura, mismos que tienen una clara interrelación con la educación y el desarrollo humano integral.

Siendo el ser humano un ser activo por naturaleza, dentro de esta amplia gama de actividades que realiza para descubrir, conocer y transformar la cultura, se va a profundizar en el juego, comprendido como una constituyente del patrimonio cultural intangible y justificando este artículo desde la importancia de recopilar las variables en las características que del juego se desprenden con el paso del tiempo, ya que el juego como otros elementos del patrimonio cultural, también sufre transformaciones constantes. Tal y como lo afirman Chang, Cerdas, Herrera, Perera y Troyo (2010), el patrimonio cultural "aunque nos viene del pasado y nos remite a nuestras raíces históricas, está en constante proceso de renovación y resignificación: se compone de aquellos bienes que a lo largo de la historia fueron 
creando quienes habitaron nuestro territorio, así como lo que en el presente seguimos creando a quienes vivimos en él" (p. 18).

Por otra parte, esta investigación desarrolla una acción en función del resguardo y recuperación del patrimonio cultural intangible, específicamente el juego desde su estudio en las regiones señaladas en esta investigación. La conservación de este legado es considerada por Chang et al. (2010) como necesaria de ser preservada principalmente porque "los bienes no materiales por su condición de intangibles han sido en la mayor parte de los casos "invisibilizados" del reconocimiento y valoración colectiva... los juegos de palmadas, rondas y adivinanzas de la infancia”... (p. 2).

Con el propósito de construir estas memorias desde un contexto concreto, se desarrolló el proyecto de investigación que engloba este artículo, el cual reconstruirá una memoria colectiva de juego desde la experiencia de diversos grupos generacionales que contemplan edades desde niños y niñas hasta personas adultas y adultas mayores, para contar con una visión de esta línea de tiempo, relaciones y diferencias entre el juego y sus características, teniendo presente que esta forma parte del patrimonio de estas comunidades y que reconstruye constantemente la cultura.

Es importante señalar que este artículo respalda los principios planteados por la Convención para la Salvaguardia del Patrimonio Cultural Inmaterial de la UNESCO, en cuanto a la importancia de salvaguardar el patrimonio cultural inmaterial, la identificación, documentación, investigación, preservación, protección, promoción, valorización, transmisión y revitalización de este patrimonio en sus distintos aspectos.

\section{Referente teórico}

\section{Cultura y patrimonio}

La cultura se visualiza como el conjunto de rasgos, características y/o conductas cultivadas en la persona que a su vez conforma un conjunto común de conocimientos dentro de un grupo determinado. Este conjunto de rasgos, características y/o conductas es una asociación indispensable para el óptimo y natural desarrollo de las personas y un cúmulo de saberes compartidos de generación en generación, el cual va adquiriendo con el paso del tiempo cambios que obedecen a la transformación de los saberes que acompaña la evolución de las sociedades. 
En el proceso de desarrollo de las personas existen dos factores determinantes: la herencia biológica y la herencia social. Este artículo se enmarca en la herencia social, entendida como el conjunto "de ideas positivas aceptadas por la sociedad a través de la historia, con lo cual se han logrado modificaciones beneficiosas en actitudes y costumbres de los pueblos" (Jaramillo, 2009, p. 65). De esta herencia social se derivan componentes que constituyen y determinan la transformación de las sociedades, tales como la cultura, entendida como un concepto generalizado en su doble aspecto, esto es, como cultivo del ser humano y como la acumulación y transmisión del acervo de conocimientos (Cagigal, 1979).

Desde la noción antropológica del concepto de cultura según Chang et al. (2010), esta es una

creación esencialmente humana, de hombres y de mujeres de toda época y lugar del planeta, que comprende todo el quehacer y saber humano y caracteriza el estilo de vida de una sociedad; es decir, las formas de sentir, pensar y comportarse; además se aprende en la convivencia social, mediante la educación informal, no formal y formal (p. 17).

La cultura según Bonfil, citado por Chang et al. (2010), vislumbra un cúmulo "de símbolos, valores, actitudes, habilidades, conocimientos, significados, formas de comunicación y de organización social y bienes materiales que hacen posible la vida de una sociedad determinada y le permiten transformarse y reproducirse de una generación a la siguiente" (p. 18).

De esta manera, se puede afirmar que la cultura es un proceso de aprendizaje y transformación continua en el ser humano, permanente en todas sus etapas de vida y al caracterizarse el ser humano, según Ordóñez (2010), como un ser activo por naturaleza, posee la capacidad de conocer su mundo, descubrir la realidad física y cultural que le rodea y, a la vez, tiene posibilidades de conocer ese mundo para transformarlo, modificar la cultura recibida y crear nueva cultura.

La cultura de un pueblo está caracterizada tanto por su patrimonio natural como por su patrimonio cultural, este último contempla una serie de bienes culturales, tangibles (materiales) e intangibles (no materiales). Un patrimonio "es una pertenencia, que puede ser: individual o colectiva; privada o pública, natural o cultural y su ámbito puede ser local, regional, nacional, mundial" (Chang, 2010, p.5), por lo que en opinión de esta misma autora, "al heredar o adquirir una persona un patrimonio, se constituye en dueño de uno o varios bienes y sobre estos adquiere derechos y deberes" (p. 15). Según Chang et al. (2010), el juego forma parte de los elementos constitutivos del patrimonio cultural como un bien intangible. 


\section{Juego y cultura}

El juego es considerado como un fenómeno cultural. Desde las reflexiones de Huizinga, en su escrito Homo Ludens (1938), se entiende al juego como "...una forma de actividad, como una forma llena de sentido y como función social” (p. 15), diferenciada de la vida normal o real si se quiere entender de esta manera.

Además, en opinión de Gordillo, Gómez, Sánchez, Gordillo y Vicente (2011), el juego potencia la capacidad para que las personas menores se autoexpresen, se autodescubran, se autoconozcan, exploren y experimenten con sus sensaciones, movimientos y relaciones, todo lo cual favorecerá la construcción de aquello que esté relacionado con el mundo que les rodea.

Así las cosas, el juego facilita a los niños y las niñas experimentar con la realidad externa, relacionarse con otros y en general tener cierta autonomía que le está prohibida en otros ámbitos (Gordillo et al., 2011).

Para Piaget (1993, citado en Gordillo et al., 2011), el seguimiento de pautas en función del mundo externo es el ejemplo de cómo el juego contribuye al establecimiento de las estructuras mentales que permiten la regulación de estas.

En esa relación con el mundo exterior, Santacana y Serrat (2001) señalan la capacidad del juego para contextualizar las cosas y para constituirse en una herramienta promotora del aprendizaje de los diversos elementos de la cultura, en tanto sus reglas y contextos estén claramente definidos.

Acerca de la importancia que tienen los juegos en la cultura, Öfele (2008) señala que estos poseen una riqueza increíble revelada mediante su estudio en profundidad, la relación con la cultura de cada región, el momento histórico en que se juega y las personas que participan en ellos, todo lo cual favorece el conocimiento de una serie de aspectos histórico-social-culturales que ayudan a entenderlos y a vislumbrar la historia y cultura de nuestros pueblos, aspecto que es fortalecido por los juguetes empleados, tanto así que para esta autora, "la historia de los juguetes es parte de la historia de la cultura del hombre" (p. 53).

Con base en lo antes expuesto, cabe pensar que como bien señalan Gordillo et al. (2011), la importancia del juego en su relación consigo mismo y el entorno en que los niños y las niñas se encuentran inmersos es fundamental para su vida y desarrollo, reforzado por el planteamiento de Brooker y Woodhead (2013) en cuanto a que los juegos que los menores de edad realizan durante su proceso de desarrollo y crecimiento, favorecen la satisfacción de sus necesidades, las 
relaciones interpersonales, el reconocer y expresar asertivamente las tensiones, tener experiencias que hagan viables sus fantasías y asimilar y poner en práctica los aprendizajes, por lo que en opinión de Garaigordobil (2008) se podría "afirmar que estimular la actividad lúdica positiva, simbólica, constructiva, creativa y cooperativa es sinónimo de potenciar el desarrollo infantil, además de tener una función preventiva y terapéutica" (p. 19).

Al respecto, Lester y Russell (2011) son del criterio de que

la habilidad de los niños para encontrar tiempo y espacio para jugar se ve afectada por una serie de factores sociales, culturales, económicos y políticos. Entre ellos se encuentran el género, el estatus socio-económico y la discapacidad. Los adultos deben prestar atención a la creación de condiciones en las que pueda surgir el juego, para responder al derecho del niño a la provisión del juego (p. x).

Así las cosas y desde la perspectiva de estos mismos autores,

la calidad de los entornos influye en la habilidad para jugar, lamentablemente, algunos lugares comunales para jugar se visualizan como: de miedo, violencia, inaccesibilidad, limitaciones en horarios y movilidad; poco atractivos, de opresión, encarcelamiento o "pueden ser tóxicos desde el punto de vista medioambiental" y generalmente estos lugares tienen propósitos, usos y horarios definidos por los adultos (p. ix).

Agregan estos autores que el juego infantil es inherente a los menores de edad, por lo que las personas adultas "no deberían destruir los sitios propios de los niños para jugar por medio de una planificación insensible, o buscando otros objetivos (de adultos), o creando lugares y programas que segreguen a los niños y controlen su juego" (Lester y Russell, 2011, p. xi).

\section{Metodología}

Tipo de investigación: Fue descriptiva porque narra las opiniones y los aportes de los participantes, permitiendo profundizar acerca de los diversos aspectos, dimensiones o componentes de la cultura y su interrelación con el juego en el tiempo dando un sentido de entendimiento como patrimonio cultural. De igual manera, fue un estudio explicativo con un enfoque de investigación mixto, al combinar técnicas cualitativas y cuantitativas para el análisis de los datos.

Población participante: La investigación se llevó a cabo en dos cantones, Desamparados en San José y Barva de Heredia, que fueron seleccionados por contar con 
una escuela, colegio y gobierno local en disposición de participar y respetando los criterios de edad y rol que desempeñan las personas colaboradoras (padres, madres, abuelos, abuelas, estudiantes, docentes, autoridades del gobierno local, entre otras).

Específicamente se seleccionaron en cada cantón en coordinación con las instituciones educativas, un grupo de niños y niñas de tercero y sexto gradod (25 por grado y escuela) y sus respectivos padres y madres; un grupo de jóvenes de octavo, décimo y undécimo años de colegio (25 por grado y colegio), 10 docentes de primaria, 10 docentes de secundaria y al menos un representante del gobierno local, así como un grupo de adultos mayores de 50 años localizados en la comunidad y que participan en grupos organizados por la municipalidad. En total fueron 170 personas de Desamparados y 158 de Barva, tal y como lo muestra la tabla 1 que se presenta a continuación.

Tabla 1. Cantidad de participantes en la investigación según grupo y cantón

\begin{tabular}{|l|c|c|}
\hline \multicolumn{1}{|c|}{ Participantes } & Desamparados & Barva \\
\hline (I ciclo) & 25 & 25 \\
\hline (II ciclo) & 25 & 25 \\
\hline (III ciclo) & 25 & 25 \\
\hline (IV ciclo) & 25 & 25 \\
\hline Familia & 25 & 18 \\
\hline Docentes & 20 & 18 \\
\hline Adultos mayores & 25 & 22 \\
\hline \multicolumn{1}{c|}{ Total } & $\mathbf{1 7 0}$ & $\mathbf{1 5 8}$ \\
\hline
\end{tabular}

Fuente: Cuestionario elaborado para las personas: estudiantes, adultas mayores, la familia y el personal docente.

Instrumentos: Se utilizaron como instrumentos cuestionarios dirigidos a las personas según grupos generacionales, estos fueron validados por profesionales y piloteados con un grupo de personas diferentes a los participantes de la investigación, que cumplían con las mismas características de edad y rol que desempeñan en el ámbito familiar y social. Los cuestionarios contenían ítems comunes, los cuales podrán ser utilizados en todos los cuestionarios que serían aplicados.

Cabe señalar que para los adultos mayores de 50 años se procedió a diseñar un taller lúdico creativo. Durante la realización de este, se invitó a los participantes a llenar el cuestionario y a disfrutar simultáneamente de una tarde de alegría, recuerdos, conversaciones y colores que les evocaran los juegos de antaño y sus experiencias construidas alrededor de estos. 
Análisis de datos: Como primer paso para el análisis de los datos, se digitalizaron las respuestas de los cuestionarios; posteriormente, se procedió a construir categorías emergentes para elaborar los gráficos y las tablas ubicando las respuestas en las categorías identificadas y realizando el conteo de frecuencias y porcentajes acompañándolos de las respectivas descripciones y análisis.

\section{Análisis de resultados}

En el análisis de datos se intentó realizar un acercamiento al pensamiento intergeneracional acerca de los temas tratados en esta investigación, por lo que las investigadoras seleccionaron algunas de las principales características con el fin de generar esos puntos de encuentro, es decir, se determinaron para este análisis comparativo los siguientes temas acerca del juego: conceptualización, importancia, valores y características como lugares, personas que acompañan y tipos de juegos. Cabe señalar que se tomaron las tres primeras categorías construidas para cada grupo.

La tabla 2 muestra los datos con el formato descrito y con la información correspondiente, a saber, la conceptualización e importancia del juego.

Tabla 2. Comparación del concepto de juego e importancia desde la visión de cada grupo generacional

\begin{tabular}{|c|c|c|c|c|}
\hline & \multicolumn{2}{|c|}{ Conceptualización del juego } & \multicolumn{2}{|c|}{ Importancia del juego } \\
\hline & Desamparados & Barva & Desamparados & Barva \\
\hline (I ciclo) & $\begin{array}{l}\text { Diversión } \\
\text { Interacción } \\
\text { Importante }\end{array}$ & $\begin{array}{l}\text { Diversión } \\
\text { Interacción } \\
\text { Entretenimiento }\end{array}$ & $\begin{array}{l}\text { Divierte } \\
\text { Salud } \\
\text { Valores }\end{array}$ & $\begin{array}{l}\text { Divierte } \\
\text { Derecho } \\
\text { No aburrirse }\end{array}$ \\
\hline (II ciclo) & $\begin{array}{l}\text { Diversión } \\
\text { Desarrollo/aprend. } \\
\text { Interacción }\end{array}$ & $\begin{array}{l}\text { Diversión } \\
\text { Interacción } \\
\text { Entretenimiento }\end{array}$ & $\begin{array}{l}\text { Aprend/diversión } \\
\text { Desestrés } \\
\text { Salud }\end{array}$ & $\begin{array}{l}\text { Disfrute/diver. } \\
\text { Derecho } \\
\text { Importancia }\end{array}$ \\
\hline (III ciclo) & $\begin{array}{l}\text { Diversión } \\
\text { Recreación } \\
\text { Entretenimiento }\end{array}$ & $\begin{array}{l}\text { Diversión } \\
\text { Interacción } \\
\text { Entretenimiento }\end{array}$ & $\begin{array}{l}\text { Desestrés } \\
\text { Aprendizaje } \\
\text { Derecho } \\
\end{array}$ & $\begin{array}{l}\text { Interacción } \\
\text { Aprendizaje } \\
\text { Distracción }\end{array}$ \\
\hline (IV ciclo) & $\begin{array}{l}\text { Diversión } \\
\text { Recreación } \\
\text { Entretenimiento }\end{array}$ & $\begin{array}{l}\text { Diversión } \\
\text { Interacción } \\
\text { Entretenimiento }\end{array}$ & \begin{tabular}{|l|} 
Desarrollo \\
Desestrés \\
Valores \\
\end{tabular} & $\begin{array}{l}\text { Aleja problemas } \\
\text { Diversión } \\
\text { Aprendizaje }\end{array}$ \\
\hline Familia & $\begin{array}{l}\text { Diversión } \\
\text { Interacción } \\
\text { Recreación }\end{array}$ & $\begin{array}{l}\text { Diversión } \\
\text { Aprendizaje } \\
\text { Entretenimiento }\end{array}$ & $\begin{array}{l}\text { Desarrollo } \\
\text { Desestrés } \\
\text { Interacción }\end{array}$ & $\begin{array}{l}\text { Aprendizaje } \\
\text { Desestrés } \\
\text { Interacción }\end{array}$ \\
\hline
\end{tabular}




\begin{tabular}{|c|c|c|c|c|}
\hline & \multicolumn{2}{|c|}{ Conceptualización del juego } & \multicolumn{2}{|c|}{ Importancia del juego } \\
\hline & Desamparados & Barva & Desamparados & Barva \\
\hline Docentes & $\begin{array}{l}\text { Diversión } \\
\text { Aprend./diversión } \\
\text { Interacción } \\
\end{array}$ & $\begin{array}{l}\text { Diversión } \\
\text { Interacción } \\
\text { Aprendiz./disfrute } \\
\end{array}$ & \begin{tabular}{|l|} 
Desarrollo \\
Derecho \\
Desestrés \\
\end{tabular} & $\begin{array}{l}\text { Aprendiz./desa. } \\
\text { Divers./aprend. } \\
\text { Desestrés }\end{array}$ \\
\hline $\begin{array}{l}\text { Adultos } \\
\text { mayores }\end{array}$ & $\begin{array}{l}\text { Expresión } \\
\text { Desarr./aprendiz. } \\
\text { Diversión } \\
\end{array}$ & $\begin{array}{l}\text { Diversión } \\
\text { Aprendizaje } \\
\text { Entretenimiento }\end{array}$ & \begin{tabular}{|l|} 
Salud \\
Desarrollo \\
Desestrés \\
\end{tabular} & $\begin{array}{l}\text { Distracción } \\
\text { Desestrés } \\
\text { Recreación }\end{array}$ \\
\hline Totales & \multicolumn{2}{|c|}{$\begin{array}{l}\text { Diversión } 14 \\
\text { Interacción } 9 \\
\text { Entretenimiento } 8 \\
\text { Desarrollo/aprendizaje } 6 \\
\text { Recreación } 3 \\
\text { Expresión } 1 \\
\text { Importante } 1\end{array}$} & \multicolumn{2}{|c|}{$\begin{array}{l}\text { Desestrés/recreación/distracción } 15 \\
\text { Desarrollo/aprendizaje } 10 \\
\text { Diversión } 4 \\
\text { Derecho } 4 \\
\text { Interacción } 3 \\
\text { Salud } 3 \\
\text { Valores } 2\end{array}$} \\
\hline
\end{tabular}

Fuente: Cuestionario elaborado para las personas estudiantes, adultas mayores, la familia y el personal docente.

Tal y como se observa en la tabla anterior, el juego es descrito mayoritariamente como una actividad que genera diversión y, por lo tanto, placer, al brindar la posibilidad de interactuar con otros y de entretenerse. Además, se menciona que el juego promueve el desarrollo integral, es decir, de las áreas psicomotora, socioemocional, cognitivo lingüística, en este sentido se señala que se logran aprendizajes valiosos y sobre todo divertidos en las personas. Otra de las acepciones que se hacen a este término tiene que ver con la recreación y expresión, se comenta que el juego es una posibilidad de recrearse y distraerse de las múltiples situaciones cotidianas, permitiendo en las personas la expresión de lo que sienten y piensan.

$\mathrm{Al}$ analizar los datos referidos a la importancia del juego y luego de conocer que casi la totalidad de los participantes atribuyen gran trascendencia a la actividad lúdica, es interesante que las justificaciones al respecto recaen en que permite el desestresarse, recrearse, distraerse, alejarse de los problemas, de las drogas y de los vicios, entre otros. Esto es que genera períodos de distensión, relajación y sirve como válvula de escape ante las situaciones que se presentan en la sociedad y que aquejan a las personas. Como valor agregado, se reafirma que el juego es una acción que deriva en aprendizajes y desarrollo integral en cada una de las áreas como se mencionó en párrafos anteriores, por lo que se podría decir que el juego no es simplemente un medio para la transmisión de contenidos recreativos, sino que tiene un papel trascendental en el desarrollo de la personalidad y el desarrollo integral de las personas. Desde esta perspectiva, el juego fomenta entre otros, la construcción y adquisición de valores y constructos de conocimiento. 
El reconocer que la actividad lúdica es un derecho y que es valiosa no solo para los niños y las niñas, sino para todas las personas, es un norte necesario de perseguir, con el fin de crear ambientes sociales más agradables y considerados con las personas que los habitan, no es por ello despreciable que se mencione que el juego promueve salud integral y valores, ya que durante la actividad lúdica las personas viven intensamente, saltan, conversan, ríen, gozan, comparten, aman, pierden, abrazan y muchas otras acciones necesarias de promover en los seres humanos con el fin de contrarrestar el modelo acelerado e individualista que prevalece actualmente en la sociedad.

Lo anterior hace pensar que el juego como un derecho potencia y promueve el cumplimiento de otros derechos en las personas.

Tabla 3. Valores que se ponen en práctica durante el juego, por grupo participante y cantón

\begin{tabular}{|c|c|c|}
\hline Grupo participante & Desamparados & Barva \\
\hline (I ciclo) & \begin{tabular}{|l|} 
Respeto \\
Amor \\
Solidaridad
\end{tabular} & \begin{tabular}{|l|} 
Respeto \\
Amistad \\
Solidaridad
\end{tabular} \\
\hline (II ciclo) & \begin{tabular}{|l|} 
Respeto \\
Solidaridad \\
Amistad \\
\end{tabular} & \begin{tabular}{|l|} 
Respeto \\
Amistad \\
Solidaridad \\
\end{tabular} \\
\hline (III ciclo) & \begin{tabular}{|l|} 
Respeto \\
Solidaridad \\
Amistad
\end{tabular} & $\begin{array}{l}\text { Respeto } \\
\text { Amistad } \\
\text { Compartir }\end{array}$ \\
\hline (IV ciclo) & \begin{tabular}{|l|} 
Respeto \\
Paciencia \\
Solidaridad
\end{tabular} & \begin{tabular}{|l} 
Respeto \\
Solidaridad \\
Honestidad
\end{tabular} \\
\hline Familia & \begin{tabular}{|l|} 
Honestidad \\
Respeto \\
Compartir \\
\end{tabular} & \begin{tabular}{|l|} 
Respeto \\
Solidaridad \\
Compartir \\
\end{tabular} \\
\hline Docentes & $\begin{array}{l}\text { Honestidad } \\
\text { Respeto } \\
\text { Solidaridad }\end{array}$ & \begin{tabular}{|l|} 
Respeto \\
Paciencia \\
Solidaridad
\end{tabular} \\
\hline Adultos mayores & \begin{tabular}{|l|} 
Respeto \\
Honestidad \\
Compartir
\end{tabular} & $\begin{array}{l}\text { Amistad } \\
\text { Respeto } \\
\text { Amistad }\end{array}$ \\
\hline
\end{tabular}




\begin{tabular}{|c|l|c|}
\hline \multicolumn{1}{|c|}{ Grupo participante } & \multicolumn{1}{|c|}{ Desamparados } & Barva \\
\hline & Respeto 14 \\
& Solidaridad 10 & \\
Totales & Amistad 7 & \\
& Compartir 4 \\
& Honestidad 3 \\
& Paciencia 2 \\
& Amor 1 \\
\hline
\end{tabular}

Fuente: Cuestionario elaborado para las personas estudiantes, adultas mayores, la familia y el personal docente.

Cuando se consulta a los grupos de informantes de esta investigación acerca de si consideran que durante el juego se ponen en práctica los valores, son enfáticos en responder que sí se da, señalando una lista amplia de estos valores. No obstante, en la tabla anterior se priorizan los tres primeros indicados por cada grupo de población, evidenciándose que para ellos los principales son: el respeto, solidaridad, amistad, compartir, honestidad, paciencia y el amor. Cabe señalar que el respeto es el que ocupa el puesto número uno, puesto que todos los grupos lo mencionan y solo en tres casos no aparece de primero en la lista. Este es un valor considerado en crisis, ya que se escucha en diferentes ámbitos comentarios acerca de que ya los niños y adolescentes no son respetuosos y que cada día se hace más difícil encontrar personas con este valor como característica, igual podríamos decir con respecto a la solidaridad y la honestidad. El punto es que si todos estamos de acuerdo en que es posible aprenderlos, aplicarlos y practicarlos por medio del juego, tenemos en relación con lo anterior, el reto de propiciar espacios para el juego y para modelar en las personas los valores, las costumbres y tradiciones que configuran la cultura y, por ende, el legado cultural. Desde la literatura se les atribuye a los valores connotaciones culturales en la medida que son representaciones simbólicas emanadas de experiencias culturales cuya existencia es inmaterial y que se transfieren de generación en generación (Mendes, citado por Espinoza, 2006) y por medio del juego como actividad primordial y globalizadora de los procesos que conforman al ser humano. Asimismo, se visualiza el juego como un espacio de expresión, de componentes racionales, emocionales e incluso físicos, que favorecen la formación de valores (Torres, Padrón y Cristalino, 2007).

Al igual que el juego los valores son representaciones simbólicas emanadas de las experiencias culturales y cuya existencia es inmaterial y su transferencia remite a las generaciones (Mendes, 2002, citado por Espinoza, 2006). Este carácter cultural intangible atribuido al juego y a los valores los hace estar en constante transformación y uno influye a los otros. 
Además, es necesario recordar que es durante el proceso de socialización cuando las personas menores de edad interiorizan, edifican los valores, momento que coincide en las épocas por excelencia atribuidas al juego. Esta característica socializadora del juego fomenta que los niños y las niñas identifiquen los aspectos de la vida que les proporcionen satisfacción a los propios intereses y que no perjudiquen a otras personas, les facilita oportunidades para la convivencia y para la identificación de actitudes que bien podrían ser aprobadas o rechazadas por los protagonistas del juego, contribuye al aprendizaje de cuáles pueden ser reforzados o evitados y representar y comprender situaciones que posteriormente pueden ser traducidas en valores.

Torres, Padrón y Cristalino (2007) plantean una relación entre el juego y los valores, en la que se destaca que el juego como espacio de expresión, de componentes racionales, emocionales e incluso físicos, favorece la formación de valores, fomenta la participación, el protagonismo, las relaciones, el ejercicio de roles, enriquece y propicia el desarrollo de experiencias de sociabilización y convivencia, que colaboran en el proceso de aprendizaje.

Tabla 4. Características del juego intergeneracional

\begin{tabular}{|c|c|c|c|c|}
\hline & \multicolumn{2}{|c|}{$\begin{array}{c}\text { ¿Dónde juegan? } \\
\text { (Lugares para jugar) }\end{array}$} & \multicolumn{2}{|c|}{$\begin{array}{c}\text { ¿Con quién juegan? } \\
\text { (Compañeros de juego) }\end{array}$} \\
\hline & Desamparados & Barva & Desamparados & Barva \\
\hline (I ciclo) & $\begin{array}{l}\text { Casa } \\
\text { Parque, plaza } \\
\text { Barrio } \\
\end{array}$ & $\begin{array}{l}\text { Parque, plaza } \\
\text { Casa } \\
\text { Centro educativo }\end{array}$ & \begin{tabular}{|l} 
Figuras parentales \\
Primos \\
Compañeros \\
\end{tabular} & $\begin{array}{l}\text { Figuras parentales } \\
\text { Primos } \\
\text { Compañeros } \\
\end{array}$ \\
\hline (II ciclo) & \begin{tabular}{|l|} 
Casa \\
Centro educativo \\
Barrio \\
\end{tabular} & \begin{tabular}{|l|} 
Casa \\
Centro educativo \\
Parque, plaza \\
\end{tabular} & \begin{tabular}{|l} 
Hermanos \\
Compañeros \\
Amigos \\
\end{tabular} & \begin{tabular}{|l|} 
Compañeros \\
Figuras parentales \\
Hermanos \\
\end{tabular} \\
\hline (III ciclo) & $\begin{array}{l}\text { Parque, plaza } \\
\text { Casa } \\
\text { Otras casas }\end{array}$ & $\begin{array}{l}\text { Casa } \\
\text { Parque, plaza } \\
\text { Barrio } \\
\end{array}$ & \begin{tabular}{|l} 
Hermanos \\
Primos \\
Compañeros
\end{tabular} & $\begin{array}{l}\text { Compañeros } \\
\text { Hermanos } \\
\text { Primos }\end{array}$ \\
\hline (IV ciclo) & $\begin{array}{l}\text { Plaza, parque } \\
\text { Centro educativo } \\
\text { Casa }\end{array}$ & $\begin{array}{l}\text { Parque, plaza } \\
\text { Casa } \\
\text { Centro educativo }\end{array}$ & \begin{tabular}{|l} 
Amigos \\
Compañeros \\
Hermanos \\
\end{tabular} & $\begin{array}{l}\text { Hermanos } \\
\text { Primos } \\
\text { Compañeros }\end{array}$ \\
\hline Familia & $\begin{array}{l}\text { Parque, plaza } \\
\text { Barrio } \\
\text { Casa } \\
\end{array}$ & \begin{tabular}{|l} 
Casa \\
Centro educativo \\
Parque, plaza \\
\end{tabular} & \begin{tabular}{|l|} 
Compañeros \\
Hermanos \\
Primos \\
\end{tabular} & \begin{tabular}{|l} 
Amigos \\
Hermanos \\
Primos \\
\end{tabular} \\
\hline Docentes & \begin{tabular}{|l|} 
Barrio \\
Casa \\
Centro educativo
\end{tabular} & $\begin{array}{l}\text { Barrio } \\
\text { Casa } \\
\text { Centro educativo } \\
\end{array}$ & \begin{tabular}{|l} 
Hermanos \\
Amigos \\
Primos \\
\end{tabular} & $\begin{array}{l}\text { Amigos } \\
\text { Compañeros } \\
\text { Hermanos } \\
\end{array}$ \\
\hline
\end{tabular}




\begin{tabular}{|l|l|l|l|l|}
\cline { 2 - 5 } \multicolumn{1}{c|}{} & \multicolumn{2}{c|}{$\begin{array}{c}\text { ¿Dónde juegan? } \\
\text { (Lugares para jugar) }\end{array}$} & \multicolumn{2}{c|}{$\begin{array}{c}\text { ¿Con quién juegan? } \\
\text { (Compañeros de juego) }\end{array}$} \\
\cline { 2 - 5 } & Desamparados & \multicolumn{1}{c|}{ Barva } & \multicolumn{1}{c|}{ Desamparados } & \multicolumn{1}{c|}{ Barva } \\
\hline \multirow{2}{*}{$\begin{array}{l}\text { Adultos } \\
\text { mayores }\end{array}$} & $\begin{array}{l}\text { Casa } \\
\text { Centro educativo } \\
\text { Barrio }\end{array}$ & $\begin{array}{l}\text { Parque, plaza } \\
\text { Centro educativo } \\
\text { Casa }\end{array}$ & $\begin{array}{l}\text { Primos } \\
\text { Amigos } \\
\text { Compañeros }\end{array}$ & $\begin{array}{l}\text { Compañeros } \\
\text { Amigos } \\
\text { Hermanos }\end{array}$ \\
\hline
\end{tabular}

Fuente: Cuestionario elaborado para las personas estudiantes, adultas mayores, la familia y el personal docente.

Se presentan en la tabla 4 dos de las características del juego que se consideran más relevantes para este artículo, a saber: lugares para jugar y personas que acompañan los espacios lúdicos. Para iniciar, cabe señalar que los lugares más señalados como alternativas de juego son la casa, el centro educativo y los espacios al aire libre públicos, por ejemplo, las plazas, parques, canchas, play ground, entre otros. Salvo pocas excepciones, estos son los lugares predilectos para las actividades lúdicas, a pesar de que tanto por la vivencia personal como por lo señalado en la literatura, se sabe que se puede jugar en cualquier lugar, siempre y cuando sea un lugar acorde con el crecimiento del niño o la niña y ser garante que se juega sin peligro alguno. La literatura señala que los espacios de juego públicos deben contribuir a mitigar efectos propios del hacinamiento y la falta de espacios para jugar en los hogares, confluyendo en actividades lúdicas significativas y los espacios de juego adecuados, en pro del fortalecimiento del desarrollo físico, psicológico y social de los educandos (Rematal, 2006).

No obstante, Rissotto y Tonucci (2002), Thomas y Hocking (2003), Kytta (2004), Karsten y van Vliet (2006) y Kinoshita (2008), citados por Lester y Russell (2011), "apuntan a la creciente institucionalización del tiempo y espacio de los niños, con la consecuente reducción del acceso independiente de los niños a los espacios públicos" (p. ix), esto deriva en "la disminución del juego al aire libre y el aumento de la supervisión adulta" (Lester y Russell (2011), citando a Karsten (2005), van Gils y otros (2009), pp. ix-x), es decir, cada vez hay menos posibilidades para jugar al aire libre y cada vez se hace necesaria una constante supervisión de las personas adultas durante el juego en estos espacios.

Desde la perspectiva de Lester y Russell, 2011, "la calidad de los entornos influye en la habilidad para jugar" (p. ix), lamentablemente, algunos lugares comunales para jugar se visualizan como: de miedo, violencia, inaccesibilidad, limitaciones en horarios y movilidad; poco atractivos, de opresión, encarcelamiento o "pueden ser tóxicos desde el punto de vista medioambiental" (p. ix) y generalmente estos lugares tienen propósitos, usos y horarios definidos por los adultos. 
En la actualidad en Costa Rica, por ejemplo, se ha visto una creciente tendencia de remodelar los parques públicos primando la estética adultista con diseños que excluyen el juego de los niños, los jardines muy cuidados, lámparas costosas y "finas", entre otros, no hacen más que desplazar a los niños de este espacio tan importante años atrás, donde las familias visitaban estos entornos para jugar y gozar de actividades lúdicas en las tardes soleadas. En este sentido se reitera la necesidad de establecer las prioridades que conllevan a contar con zonas de placer y juego.

En la tabla 4, se puede observar que las personas que acompañan la actividad de juego de los niños y lsd niñas en los primeros años de vida fueron figuras parentales, no obstante, las otras poblaciones no los mencionan. Esto es coherente con lo citado en la literatura, donde se comenta que el primer contexto para el juego es la relación madre e hijo, seguido del mundo y la escuela como contextos adicionales, ya que "el clima lúdico va a estar presente en la relación de aprendizaje en el ámbito escolar" (Rematal, 2006, p. 15). Sin embargo, pareciera que la escuela y los actores adultos les quedan debiendo a los niños y las niñas en ese acompañamiento durante los períodos de juego, ya que únicamente se contempla a los pares o compañeros como jugadores. Desde la visión de Mussen, Conger y Kagan, citados en García y Rubio (2011), las personas adultas y significativas tienen la responsabilidad de brindar a los niños y las niñas, experiencias que propicien su desarrollo físico, psíquico y social; potenciando la interacción con otros, en contextos más amplios, como la comunidad o la escuela.

En este sentido, es importante apuntar que el contexto familiar es uno de los principales espacios de y para el juego (Minuchin y Fishman, 2004), al ser la familia el grupo con un parentesco, costumbres, hábitos similares y al habitar un mismo espacio, comparten afectos, preocupaciones, tareas y responsabilidades, entre otros, que generan en esta dinámica unas pautas de interacción que influyen en el comportamiento de los miembros del núcleo familiar, definiendo las conductas, facilitando la convivencia y el sentido de pertenencia al grupo familiar (Minuchin y Fishman, 2004).

Asimismo, los ámbitos educativos como espacios de juego, específicamente las escuelas

forman parte del contexto social y cultural al que pertenecen los alumnos y al mismo tiempo forman parte de un contexto más amplio que las integra a un proyecto educativo de carácter nacional. Es decir, tienen saberes y prácticas cercanas a la cultura propia del lugar y también deben sostener propósitos que son diversos y más amplios a través de estas acciones y pautas comunes los alumnos se acercan en términos de desarrollo cultural, cognitivo y social (Valiño, 2005, p. 2). 
Señalando lo anterior, es importante resaltar la función de la familia, la comunidad y el contexto educativo como principales responsables de la garantía del derecho al juego no solo para las personas menores de edad sino para todos los habitantes.

Tabla 5. Juegos preferidos por los grupos participantes según cantón

\begin{tabular}{|l|c|c|}
\hline \multicolumn{1}{|c|}{ Grupo participante } & Desamparados & Barva \\
\hline (I ciclo) & $\begin{array}{c}\text { Juego al aire libre } \\
\text { Videojuegos }\end{array}$ & $\begin{array}{c}\text { Juego al aire libre } \\
\text { Juegos de imitación }\end{array}$ \\
\hline (II ciclo) & $\begin{array}{c}\text { Juego al aire libre } \\
\text { Videojuegos }\end{array}$ & $\begin{array}{c}\text { Videojuegos } \\
\text { Juego al aire libre }\end{array}$ \\
\hline (III ciclo) & $\begin{array}{c}\text { Juego al aire libre } \\
\text { Videojuegos }\end{array}$ & $\begin{array}{c}\text { Juego al aire libre } \\
\text { Juegos de mesa }\end{array}$ \\
\hline (IV ciclo) & $\begin{array}{c}\text { Juego al aire libre } \\
\text { Videojuegos }\end{array}$ & $\begin{array}{c}\text { Juego al aire libre } \\
\text { Juegos de mesa }\end{array}$ \\
\hline Familia & $\begin{array}{c}\text { Juegos de mesa } \\
\text { Juego al aire libre }\end{array}$ & $\begin{array}{c}\text { Juego al aire libre } \\
\text { Juegos de mesa }\end{array}$ \\
\hline Docentes & $\begin{array}{c}\text { Juegos de mesa } \\
\text { Videojuegos }\end{array}$ & $\begin{array}{c}\text { Juegos de mesa } \\
\text { Juego al aire libre }\end{array}$ \\
\hline Adultos mayores & $\begin{array}{c}\text { Juegos de mesa } \\
\text { Juego al aire libre }\end{array}$ & $\begin{array}{c}\text { Juegos de mesa } \\
\text { Otros }\end{array}$ \\
\hline
\end{tabular}

Fuente: Cuestionario elaborado para las personas estudiantes, adultas mayores, la familia y el personal docente.

Con respecto a los juegos preferidos, se puede observar en la tabla anterior, que los privilegiados por las personas son los que se llevan a cabo al aire libre, ya que excepto en una ocasión, siempre se mencionan en las dos primeras categorías mostradas en la tabla. Se puede decir que a pesar de que la tecnología ha permeado los espacios y prácticas de juego de los niños y las niñas, todas las poblaciones priorizan el hacer actividad lúdica en espacios exteriores donde la libertad y el contacto con la naturaleza son posibles.

Cabe señalar que las personas de Desamparados comparativamente con las de Barva ubican con mayor frecuencia los videojuegos en lugar de predilección y en el caso de Barva solo los niños y las niñas de segundo grado los ven como sus preferidos. Este hallazgo llama la atención debido a que posiblemente en un lugar urbano con el cantón herediano, los menores de edad aún tienen mayores posibilidades de jugar al aire libre que los de la zona urbana de Desamparados. 


\section{Conclusiones}

- $\quad$ El juego es definido como diversión, entretenimiento, disfrute, alegría, promueve la convivencia, el movimiento, la libertad, el compartir, el desestrés y el reír mencionando pocas veces al juego como un derecho.

- Se visualiza el juego como una actividad casi exclusivamente de y para los niños y las niñas, con pocas excepciones mencionan a las personas adultas o adultas mayores como promotoras y protagonistas de esta actividad.

- Es interesante resaltar que se hace constante alusión a que el juego tiene un tiempo y un espacio preestablecidos, es decir, debe darse en un "momento apropiado", lo cual hace pensar en las significativas limitaciones que enfrentan hoy la mayoría de los niños y las niñas al jugar.

- Casi para la totalidad de los participantes el juego es de suma importancia, en razón de que promueve el desarrollo integral, así como el aprendizaje de conceptos y esencialmente los valores.

- $\quad$ El juego es defendido como un derecho que provoca estados de alegría y la risa, considerados necesarios para obtener la felicidad, aunque no por todos los grupos participantes.

- Se nota en algunas de las respuestas ofrecidas que "el estudio", la disciplina y obediencia siguen estando por encima de las posibilidades de jugar, siendo esta la actividad primordial de aprendizaje y desarrollo integral, y obviamente de contacto con su cultura.

- Los pares escolares son los principales compañeros de juego, a su vez, la familia desarrolla un rol importante en la actividad que de esta índole desarrollan los menores de edad. Los maestros juegan en menor proporción con los estudiantes, a pesar de que en los ambientes escolares deberían incorporarse cotidianamente en el juego que constantemente se realice, en virtud de que es indiscutible que es un óptimo medio para aprender.

- Es necesario concienciar a las personas adultas responsables de la crianza de las personas menores de edad, acerca de que los juegos que practican los niños y las niñas que viven en este país, permiten compartir rasgos identificadores, integradores y representativos de la cultura costarricense, por lo tanto, se convierten en el patrimonio intangible, el legado y la herencia de nuestras generaciones hacia ellos y ellas, todo lo cual hace indiscutible el valor que tiene durante el proceso de crecimiento y desarrollo de la persona, en todo su ciclo de vida.

- Diseñar entornos de calidad para el juego intergeneracional que sean seguros, cálidos, accesibles, libres de violencia, con horarios flexibles, atractivos, pensados por y para permitir el juego de todas las personas, indistintamente de sus edades. 
- $\quad$ Diseñar espacios para el juego al aire libre que no desplacen a los niños y las niñas para que practiquen sus actividades lúdicas sin temor a ser castigados por cuidar la estética establecida desde el mundo adulto.

\section{Referencias}

Araya, A., Castro, M. y Morales, M. E. (2015). El juego como patrimonio cultural: percepción de las personas de diferentes generaciones en los cantones de Desamparados y Barva. Informe de investigación. San José, Costa Rica: Universidad de Costa Rica y Universidad Nacional.

Brooker, L. y Woodhead, M. (2013). El derecho al juego. La primera Infancia en Perspectiva 9. The Open University. Reino Unido: Cambrian Printers.

Cagigal, J. M. (1979). Cultura intelectual y cultura física. Buenos Aires, Argentina: Editorial KAPELUSZ S.A.

Chang V., G.; Cerdas A., A. L.; Herrera G., M.; Perera D., I. A. y Troyo, V. E. (2010). Patrimonio Cultural: diversidad en nuestra creación y herencia. San José, Costa Rica: Ministerio de Cultura, Juventud y Deportes. Centro de investigación y conservación del patrimonio cultural.

Chang V., G. (2010) Patrimonio Cultural: Bienes Materiales e Intangibles que nos identifican. En: Chang V., G.; Cerda A., A. L.; Herrera G., M.; Perera D., I. A. y Troyo V. E. (2010). Patrimonio Cultural diversidad en nuestra creación y herencia. San José, Costa Rica: Ministerio de Cultura, Juventud y Deportes. Centro de investigación y conservación del patrimonio cultural.

Espinoza, A. (2006). Estrategias para fortalecer los valores en educación básica. Propuesta didáctica. Curso estatal de actualización para la XV etapa de carrera magisterial. San Luis Potosí. México. Recuperado de https://www.academia.edu/6664914/Espinosa_H._A._ Ren\%C3\%A9._2006_._Estrategias_para_fortalecer_los_valores_en_educaci\%C3\%B3n_ b\%C3\%A1sica._Propuesta_did\%C3\%A1ctica

García, M. y Rubio, A. (2011). El juego y las relaciones sociales en niños de preescolar. Tesis para obtener el título de Licenciatura en Psicología. Universidad Michoacana de San Nicolás Hidalgo.

Garaigordobil, M. (2008). Importancia del juego infantil en el desarrollo humano. En: Bañeres, D.; Bishop, A.; Cardona, M. C.; Comas, O.; Escuela Infantil Platero; Garaigordobil, M.; Hernández, T.; Lobo, E.; Marrón, M. J.; Ortí, J.; Pubill, B.; Ruiz de V., A.; Soler, M. P. y Vida, T. El juego como estrategia didáctica. Claves para la innovación educativa. Barcelona, España: Editorial Graó.

Garre, F., Sanguinetti, M. y Ramos, A. (1997). Juegos infantiles tradicionales. Patrimonio cultural que se pierde... Recuperado de: http://www.oocities.org/emuseoros/Docs/juegos_iberoam.html 
Gordillo, M., Gómez, M.; Sánchez, S.; Gordillo, T. y Vicente, F. (2011). El juego infantil en un mundo de cambio. Universidad de Extremadura. Badajoz. Desafíos y perspectivas actuales de la psicología en el mundo de la infancia. International Journal of Developmental and Educational Psychology. INFAD. Revista de Psicología, 1(1). Pp. 197-206.

Huizinga, J. (1938). Homo Ludens. Buenos Aires, Argentina: Emecé Editores S.A.

Jaramillo A., J. (2009). La cultura contra el mundo. San José, Costa Rica: EUNED.

Lester, S. y Russell, W. (2011). El derecho de los niños y las niñas a jugar: Análisis de la importancia del juego en las vidas de niños y niñas de todo el mundo. Cuadernos sobre Desarrollo Infantil Temprano 57s. La Haya, Países Bajos: Fundación Bernard van Leer. Recuperado de http://www.de0a18.net/pdf/doc_drets_5_elderecho.pdf

Minuchin, S. y Fishman, C. (2004). Técnicas en terapia familiar. (1ª . Edición). Argentina: Paidós.

Öfele, M. (2008). Repensar la niñez en el siglo XXI. Los espacios del juego y del jugar en la sociedad actual. Congreso de la Red Universitaria de Educación Infantil. República de Argentina. Septiembre 2008. Recuperado de http://reduei.com/wp-content/uploads/2012/06/ Espaciosdeljuego-Draay57157.pdf

Ordóñez, J. (2010). Introducción a la Pedagogía. San José, Costa Rica: EUNED.

Rematal, L. (2006). El juego como instrumento educativo y de desarrollo integral. Revista de la Pontificia Universidad Católica del Ecuador. Recuperado de http://www.puce.edu.ec/publicaciones/Centro_de_Publicaciones/Revistas/Publicaciones/Revista\%2078.pdf

Santacana, J. y Serrat, N. (2001). Una reflexión sobre conceptos históricos aprendidos a través del juego. Didáctica de las Ciencias Sociales, Geografía e Historia, OCT-DIC; VIII, 37-45.

Torres, J.; Padrón, F. y Cristalino, F. (2007). El Juego: Un espacio para la formación de valores. Omnia, vol. 13, núm. 1, enero-abril, pp. 51-78, Universidad del Zulia Venezuela. Recuperado de http://www.redalyc.org/pdf/737/73713104.pdf

Valiño, G. (2005). Encuentro Regional de Educación Inicial. "Intercambio para el fortalecimiento de políticas en educación inicial”. Ministerio de Educación, Ciencia y Tecnología. Ciudad de Buenos Aires, junio de 2006. Recuperado de http:/www.me.gov.ar/curriform/ publica/inicial_valinio.pdf

Recibido: 28/3/2017

Aceptado: 31/10/2017 